\title{
Context in Design Science Research: Taxonomy and Framework
}

\author{
Alexander Herwix \\ University of Cologne and Leipzig University \\ herwix@wiso.uni-koeln.de
}

\author{
Philipp zur Heiden \\ Paderborn University \\ philipp.zur.heiden@upb.de
}

\begin{abstract}
One of the open methodological concerns for design science research (DSR) in information systems is how to think about and deal with the notion of context. This paper takes an important step toward clarifying the notion of context and elaborates how it can be dealt with from a DSR perspective. In particular, we present a coherent theoretical account of context grounded in pragmatism. Moreover, we also reify this understanding into a context taxonomy and context framework for DSR. Altogether, we intend to provide a sound foundation and a fruitful platform for DSR that is more attuned to the particularities of context.
\end{abstract}

\section{Introduction}

Design science research (DSR) in information systems (IS) is a research paradigm striving to provide the theoretical foundation for scientifically sound, real world problem solving [1-4]. As such, DSR has an important role to play if IS researchers want to answer calls for a more responsible engagement with our world's needs [5-77]. One important and largely unresolved methodological challenge in DSR (and, indeed, IS research at large) is the question of how to think about and deal with the notion of context [8,9].

In general, the term context is used to refer to the environment or setting in which something exists [10]. In the realm of IS research, it is often understood to be the aspects of the environment that are relevant for explaining a focal phenomenon [8]. In DSR in particular, context is broadly viewed as the environment which surrounds an artifact or the source of the requirements that an artifact is to be evaluated against [2. 11]. But there is only limited agreement and a shallow conceptual understanding of what context implies for DSR in practice [8, 11].

For instance, to the best knowledge of the authors, there is no explicit and theoretically grounded discussion of context in relation to DSR to be found. At the same time, extant IS research has raised concerns about the way IS scholars deal with context [8,9]. In particular, it is observed that research is often reported in ways that may mislead about the generalizability [12 13] or projectability [14, 15] of findings [8, 9]. However, remedies for this situation are hindered by the fact that even prolific senior scholars have trouble agreeing on a common understanding of what context is and how it should be dealt with [9, 16].

We conclude that it is necessary to deepen the discussion on context in DSR if we are to bring more clarity to the IS field. We contribute toward closing this gap by unpacking what context is and how it should be dealt with in DSR. We investigate the research question: How can context in DSR be conceptualized?

We answer this question in three steps. First, we develop a coherent theoretical account of context that is grounded in Pragmatism, the philosophical core of DSR [2, 17]. Second, we present a context taxonomy [18] which clarifies a set of nine dimensions that are relevant and useful when thinking about and dealing with context. Third, we present a context framework for DSR that relates some of the main dimensions from our taxonomy with the conceptual flow of DSR (i.e., the general DSR cycle [3]). We also discuss how our results enable design science researchers to work with context more effectively and efficiently. Altogether, we contribute to the conceptual and methodological advancement of DSR but acknowledge that further evaluation and development of our results are desirable. Thus, we encourage other design science researchers to use, test or refine our results.

The remainder of our paper is structured as follows. In Section 2, we develop the theoretical background of our study. In Section 3, we outline our approach to taxonomy development. In Section 4, we present a taxonomy for context in DSR as our main finding. In Section 5, we present a context framework for DSR, before discussing the findings of our study and concluding the paper in Section 6. 


\section{Theoretical background}

Context is a concept that has been avidly discussed in IS research as well as academic research at large [8. 9. 19-26]. Etymologically the term developed from the Latin word contexere "to weave or join together" [10]. Today it generally refers to the environment or setting in which something exists [10]. Building on this understanding, contemporary research has often conceptualized context rather loosely as that which surrounds an object of interest and helps by its relevance to explain it (e.g., temporal, geographical, cultural, cognitive or emotional aspects) [8].

According to Avgerou's [8] recent and comprehensive review, this understanding of context is often informed by a distal ontological perspective- the understanding that the world is made up of interactions among stable material and social entities. Most IS research engaging with context conforms to this perspective. It can be said to embrace a mechanistic worldview, where the world is made up of things and parts that interact through forces or other forms of energy [22]. In this perspective, major challenges with regard to context arise [8,9]:

- What aspects of the environment should be included as part of the context?

- How can we evaluate whether context is adequately represented?

- How can we generalize research findings from one case to another?

This understanding of context can be contrasted to a proximal ontological perspective- the understanding that the world is an entangled whole that is in a constant state of change and evolution. In IS research this perspective is most readily associated with Actor Network Theory (ANT) [27] as well as work on sociomateriality [28] and agential realism [8, 29]. This type of research can be said to embrace a contextualistic worldview, where a phenomenon and its context form a whole which is so intertwined and entangled that it cannot readily be separated into stable entities or parts [22]. Thus, a major challenge that arises for this perspective is:

- How can we think about context in a productive manner when no ontologically real separation of phenomenon and context is presumed to be possible?

Prior work has proposed several alternatives for addressing these conflicting perspectives. First, ANT advocates for an acontextual stance, where context is simply discarded as a concept [8, 27]. ANT researchers are encouraged to follow the actors and focus on describing all of their relationships that are relevant to a focal phenomenon or research question-the concept of context loses importance because each case is understood to be unique and to be described in full [27].

Second, in agential realism, context is operationalized as "sociomaterial configurations of different scale that are constantly formed and reformed in relation to each other" [8, pp. 996-997]. This notion has been argued to be a rather abstract and vague notion in need of further explication and refinement [8].

Third, functional contextualism ${ }^{17}$ has been proposed as a pragmatic solution for transcending the dichotomy between distal and proximal perspectives [26]. In functional contextualism, any representation of context is understood to be a construction that can only be evaluated in terms of the utility that it contributes towards an analytical goal. As Zettle et al. [26] illustrate, this perspective allows for a highly coherent and effective analysis of context that is useful for working with mostly stable but also highly dynamic phenomena. It is also well-grounded in pragmatism.

Given our intent to develop an account and understanding of context for the pragmatically-oriented field of DSR [2], we adopt a position inspired by functional contextualism [26] as this framing helps us to resolve the challenges associated with the notion of context in a well-grounded and pragmatic manner.

Figure 1 illustrates our understanding. We suggest that any phenomenon always occurs in-context. By this we mean that there is no inherently true or real way in which phenomenon and context could be separated. Rather, we acknowledge that phenomenon and context could be separated in any number of ways. We need analytical goals to guide and evaluate any such separation and meaningfully operationalize context.

An analytical goal provides a frame of reference for evaluating the understanding of context in terms of the pragmatic truth criterion of successful working [22, 26] or, put differently, successfully fulfilling its function. Multiple analytical goals may co-exist and fulfill complementary functions for an actor. Altogether, we define context in a pragmatic sense as the aspects of the environment that are relevant for an actor to achieve a particular analytical goal in relation to a focal phenomenon $!^{2}$

Given this understanding, we can already readily

\footnotetext{
${ }^{1}$ Functional contextualism is a modern philosophy of science grounded in pragmatism that has been developed as the theoretical foundation for the research field of contextual behavioral science [26].

${ }^{2}$ Thus, depending on the analytical goal and the focal phenomenon, actors may or may not be part of the focal phenomenon or its context.
} 


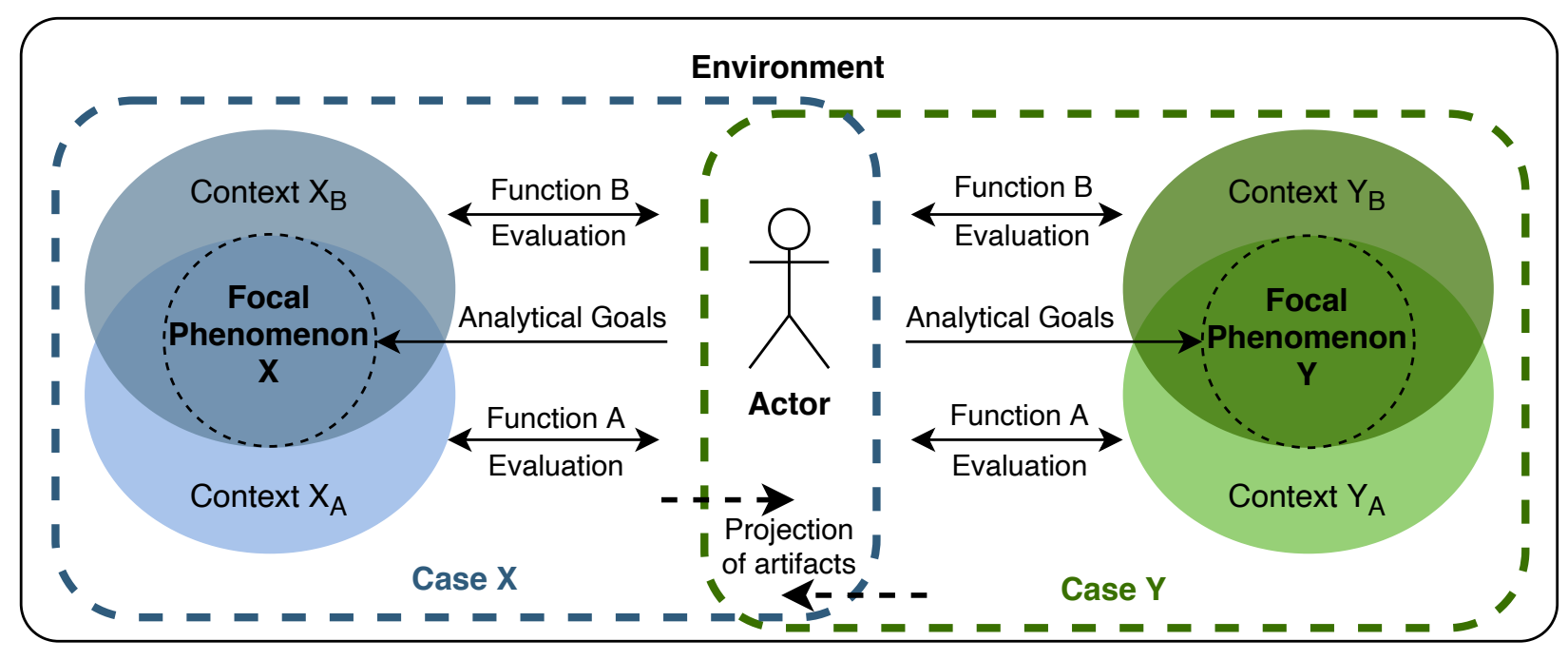

Figure 1: Illustration of our understanding of context. Any phenomenon always occurs in-context and can only be meaningfully separated from its context in relation to the analytical goals of an actor. Put differently, if an actor delineates a context it does so to fulfill a function against which it is to be evaluated. Different delineations of context are possible depending on the analytical goals of an actor and the nature of the focal phenomenon. Projection of artifacts occurs if an actor successfully reuses and adapts artifacts (e.g., conceptual models) from one case for another.

address most of the challenges regarding the notion of context raised by prior research. Table 1 shows an overview of how we address these challenges. However, we still need to elaborate how research findings can be generalized or-in the case of DSR-projected to other cases (see Figure 11). For this, we turn to the literature on projectability in DSR as well as functional contextualism for inspiration [4, 14, 15, 26].

Projectability provides an alternative to the concept of generalizability that has been argued to be more appropriate for thinking about the abstraction and reuse of knowledge with a design or action orientation [14. 15]. In short, projectability references the extent to which an artifact is able to help create intended regularities in possible but as of yet unobserved situations (i.e., other existing cases but also not yet existing possible worlds or realistic counterfactual situations) [4, 14, 15]. In other words, an artifact is more projectable if it can achieve its intended purpose in more situations [4]. Thus, projectability is an inherently pragmatic concept that facilitates thinking about the reuse and evolution of knowledge in terms of the pragmatic truth criterion of successful working [22, 26]. It aligns well with our proposed pragmatic understanding of context.

As a consequence, we suggest to discard the question of generalizability and the associated quest for universal laws that describe "reality" [12, 13]. Instead we take the pragmatic position that it is impossible to know if something is "real" or not and must acknowledge that our understanding as well as the world can change at any time. Laws which may have been thought to be real in the past may turn out to be wrong. Things that have worked in the past may not work in the future. We can only ever hope to create artifacts (e.g., conceptual models, theories, IT artifacts, etc.) that are more or less successful in achieving given analytical goals as determined by trial and error. This position has been termed an aontological stance in prior work because no ontological claims about the nature of reality are made [26].

In practical terms, we advocate for the pragmatic development and use of abstract principles [26, 30] and related artifacts that can usefully inform and support design and action in a broad variety of possible situations [4, 14, 15, 26]. Such research outcomes ought to be evaluated in terms of successful working, ideally, through rigorous empirical experimentation (e.g., randomized controlled trials or interrupted time-series experiments [31]).

\section{Research method: Taxonomy development}

Our goal for this study is to conceptualize context to help design science researchers gain a better understanding of context and become more effective and efficient at their work. After an in-depth engagement with the literature on context in IS research, we decided to approach the goal pragmatically and focus on the 
Table 1: Summary of how our account of context addresses the context challenges identified in prior research.

How can we think about context in a productive manner when no ontologically real separation of phenomenon and context is presumed to be possible?

Context is not understood to be a representation of reality but an artificial construction that should be pragmatically evaluated in terms of successful working with respect to the analytical goals.

\begin{tabular}{l}
\hline What aspects of the environment should be included as part of the context? \\
\hline The context should include all aspects that are necessary to effectively and efficiently achieve the analytical goal. \\
\hline How can we evaluate whether context is adequately represented? \\
\hline The quality of the analysis can be pragmatically assessed against its ability to contribute toward achieving the \\
analytical goal (i.e., the degree to which it achieves its function). \\
\hline How can we generalize research findings from one case to another? \\
\hline We discard the question of generalizability and instead advocate for a focus on projectability. Thus, the question we \\
are interested in is not the universality of a research finding but how successfully it can be adapted to other cases. \\
\hline
\end{tabular}

development of a context taxonomy as extant research had already demonstrated that taxonomies can be useful decision aids in design processes [32]. For this, we followed the iterative taxonomy development method by Nickerson et al. [18] as detailed in the online appendix 3 of this paper.

A taxonomy consists of $n$ dimensions each consisting of $k$ mutually exclusive and collectively exhaustive characteristics such that any phenomenon under consideration can be classified with exactly one characteristic for each dimension. In the beginning of the taxonomy development, a meta-characteristic should be defined to provide a point of reference for the identification of the characteristics of the taxonomy. To avoid "naïve empiricism", every characteristic should be a logical consequence of the meta-characteristic. Given the purpose of our taxonomy, we used our definition of context as our meta-characteristic: the aspects of the environment that are relevant to achieving particular analytical goals in relation to a focal phenomenon.

We then adopted the objective and subjective ending conditions proposed by Nickerson et al. [18] to decide when to terminate the iterative taxonomy development process (see online appendix ${ }^{3}$ ). However, we refrained from considering the objective ending condition "at least one object must be classified under every characteristic of every dimension" because we did not find empirical examples for three characteristics that were, nevertheless, strongly plausible to exist in a larger data set. Until the ending conditions were met, we conducted six iterations of both conceptual-to-empirical and empirical-to-conceptual development approaches.

For the conceptual-to-empirical development

$\sqrt[3]{\text { https://osf.io/5jkaq/ }}$ approach, we reviewed the underlying literature on DSR as well as context in IS research and related disciplines. We conducted a systematic literature review [33] by searching for the keywords context and design science in the abstracts of all articles in the senior scholars' basket of journals we could search via EbscoHost, Proquest, and AIS e-library databases. We identified 28 papers, of which we found eight to be relevant. Through backward and forward search and scanning further journals and conference proceedings, we identified 24 additional papers relevant for our conceptual-to-empirical approach.

For the empirical-to-conceptual development approach, we used the mentions of context in the abstracts of DSR papers published at DESRIST to identify and validate dimensions of our taxonomy from empirical data. We generated a data set of 78 paper abstracts with 110 mentions of context as an empirical basis for our analysis.

After six iterations of both conceptual-to-empirical and empirical-to-conceptual development approaches, all of the occurrences of context we analyzed could be grouped into exactly one of the characteristics of each dimension $(n=71)$, except those that did not correspond to the description of a context but rather referred to a different use of the term (e.g., as a definition, a keyword, or an adjective; $n=39$ ).

For the complete list of the papers used in the taxonomy development as well as the empirical data set of paper abstracts and the classifications of each context occurrence refer to the online appendix ${ }^{3}$. 


\section{A context taxonomy for design science research}

Our context taxonomy for DSR builds on our pragmatic definition of context. Thus, context can be thought of as a cognitive frame that aims to be useful for researchers. It helps to establish order and agency by decomposing an overwhelmingly complex phenomenon-in-context into a focal phenomenon, its relevant context, and the broader environment. It may be reified in the form of artifacts (e.g., conceptual models). It is at least implicitly but better explicitly assessed against analytical goals. Understanding context in this way imbues it with a clear purpose: Emphasize certain features of the environment while muting others to facilitate the achievement of analytical goals.

Against this backdrop, Table 2 presents a context taxonomy for DSR that characterizes context along nine key dimensions, which our investigation identified to be relevant when conducting DSR. Extending from our meta-characteristic, our taxonomy suggests that contexts in DSR: fulfill a specific function, have a particular focus, concern a specific scope, relate to a reference field, have a theoretical perspective, may be distinguished in their fact-orientation, historical-orientation as well as time awareness, and are associated with different levels of control.

For the dimension function, we identified three analytical goals discussed in epistemology: consensus, correspondence, and coherence [34]. We mapped these epistemological goals to corresponding functional perspectives readily associated with DSR: awareness, improvement, and integration. Awareness as a context function is concerned with the analytical goal of consensus regarding the quality of a thing (e.g., problem, strategy, or solution) and most readily associated with activities in the relevance cycle of DSR [2]. Improvement as a context function is directed at the analytical goal of correspondence between actual and desired states of the world ${ }^{4}$ and generally associated with activities in the design cycle of DSR [2]. Integration as a context function is focused on the analytical goal of coherence between contexts in the environment and often associated with activities in the rigor cycle of DSR [2]. Each characteristic was coded at least 11 times.

The dimension focus suggests that contexts can be grouped into three classes that map them to the general DSR cycle [3]: problem relating to a focus on

\footnotetext{
${ }^{4}$ We highlight that our interpretation of correspondence is inspired by pragmatism and should be distinguished from the more traditional interpretation referring to a correspondence between a claim and reality. We posit that our interpretation is more general and can accommodate the traditional interpretation as a special case.
}

problem awareness, strategy relating to the articulation of alternative design approaches in the suggestion stage, and solution relating to the development and evaluation of particular solutions. We have arrived at this categorization because it crystallizes the evolution of concerns and shifts in focus which occur as DSR progresses from problem to solution and back again [3. 35]. Intriguingly, extant research has highlighted that these shifts in focus do not only occur in individual DSR projects but also DSR streams in which multiple DSR projects culminate [3]. As such, it needs to be acknowledged that contexts can be nested along this dimension. For instance, an overarching DSR stream may be in a state where DSR projects mainly focus on strategy but any individual DSR project may still consider all three context foci. Each characteristic was coded at least 10 times.

In the dimension scope, we aim to capture the relative size of a context in relation to the environment. We recognize four different characteristics, namely local, domain, global, and universal [6]. The local scope encompasses the real world directly surrounding or immediately impacting the focal phenomenon. The domain scope encompasses practice or academic fields that bundle and integrate discourse about phenomena that are linked by shared characteristics. The global scope aims to transcend the domain scope by encompassing integrative discourse about the relationships between domains with an eye toward establishing relative priorities between them. The universal scope encompasses discourse about foundational questions that are relevant for the analysis of all phenomena, for instance, how to make judgments in terms of value or truth. We did not find any occurrence of a global scope or a universal scope in our analysis. Nevertheless, we still decided to keep them in the taxonomy as these scopes have been argued to be neglected but important scopes for impactful DSR [6].

The dimension reference field suggests that contexts may be related to extant bodies of knowledge. We propose two different characteristics-practice and academic reference fields-but more in-depth classifications may developed by future research. Each characteristic was coded at least 22 times.

The dimension perspectives articulates that different theoretical foci can guide a context analysis. We suggest three distinct characteristics-technical, social, and mix - but acknowledge that more detailed sub-classifications may need to be developed in the future to better reflect the broad range of IS research [36]. A technical perspective emphasizes the material or algorithmic features and aspects of the environment. A social perspective emphasizes the social 
Table 2: Context Taxonomy for DSR. Numbers indicate the prevalence of each characteristic in our data set $(n=71)$.

\begin{tabular}{|c|c|c|c|c|}
\hline Dimension & \multicolumn{4}{|c|}{ Characteristics } \\
\hline Function & Awareness (39) & \multicolumn{2}{|c|}{ Improvement (21) } & Integration (11) \\
\hline Focus & Problem (23) & \multicolumn{2}{|c|}{ Strategy (10) } & Solution (38) \\
\hline Scope & Local (28) & Domain (43) & Global (0) & Universal (0) \\
\hline Reference Field & \multicolumn{2}{|c|}{ Practice (49) } & \multicolumn{2}{|c|}{ Academic (22) } \\
\hline Perspective & Technical (2) & \multicolumn{2}{|c|}{ Mix (63) } & Social (6) \\
\hline Fact-orientation & \multicolumn{2}{|c|}{ Factual (45) } & \multicolumn{2}{|c|}{ Counterfactual (26) } \\
\hline Historical-orientation & Past (4) & Present (66) & Future (1) & Invariant (0) \\
\hline Time Awareness & \multicolumn{2}{|c|}{ Dynamic (55) } & \multicolumn{2}{|c|}{ Static (16) } \\
\hline Control & \multicolumn{2}{|c|}{ Naturalistic (65) } & \multicolumn{2}{|c|}{ Artificial (6) } \\
\hline
\end{tabular}

and cultural features and aspects of the environment. A mixed perspective emphasizes a sociotechnical or sociomaterial perspective on the environment in which technical as well as social features and aspects of the environment are both reasonably considered. Each characteristic was coded at least 2 times.

With the fact-orientation dimension, we highlight that a context analysis may reference an actual (factual) or a non-actual (counterfactual) state of the world [15]. Factual means that a context analysis aims to faithfully represent the actual state, whereas counterfactual analysis is concerned with non-actual (but generally possible) states of the world (i.e., projectability; see Section 2). For instance, a context analysis with a counterfactual orientation could be concerned with possible application areas for a design solution. Each characteristic was coded at least 26 times.

In the dimension historical-orientation, we emphasize that a context analysis is often temporally restricted. We identify four different characteristics to represent the main time span that a context analysis considers, i.e., past, present, future, or invariant $~_{5}^{5}$ Past means that a context analysis is mostly concerned with the historical context [37]. Present means that a context analysis is mostly concerned with the presently existing context. Future means that a context analysis is mostly concerned with the future development and context of a phenomenon. Invariant means that a context analysis is mostly concerned with time-invariant (i.e., unchanging) features of the context. Although we did not find an invariant orientation in our empirical data, we still decided to keep this characteristic in our taxonomy as it represents a reasonable and logical option.

\footnotetext{
${ }^{5}$ Note: These characteristics have somewhat fluid boundaries. For example, context analyses concerned with the present generally also concern the past and the future as time is continuously advancing.
}

The dimension time awareness highlights that time as a property of context may be more or less prominently considered. We suggests two different high-level characteristics to distinguish how time may be conceptualized in context analyses: dynamic and static. Dynamic means that a context analysis emphasizes time as property of context and considers the environment to be continuously changing and evolving. Static means that a context analysis is less concerned with time and considers the environment to be mostly static and not much changing. More detailed sub-classifications may need to be developed in the future to better reflect potential nuances within these two broad perspectives. Each characteristic was coded at least 16 times.

Finally, the characteristics of the dimension control represent the amount of control that the creator of a context analysis has over the context. We propose that different forms of control are associated with naturalistic or artificial contexts [38]. A naturalistic context concerns a natural or field environment in which control is rather limited. An artificial context concerns an artificial (e.g., simulation) or lab environment in which a larger degree of control is given. Each characteristic was coded at least 6 times.

\section{A context framework for DSR}

Our context framework for DSR shown in Figure 2 integrates key dimensions from our context taxonomy with a procedural perspective on DSR and elaborates on this interplay. In particular, the context framework combines the function, focus and scope dimensions of our context taxonomy into a three-dimensional grid of 36 context configurations that are stereotypical for DSR 


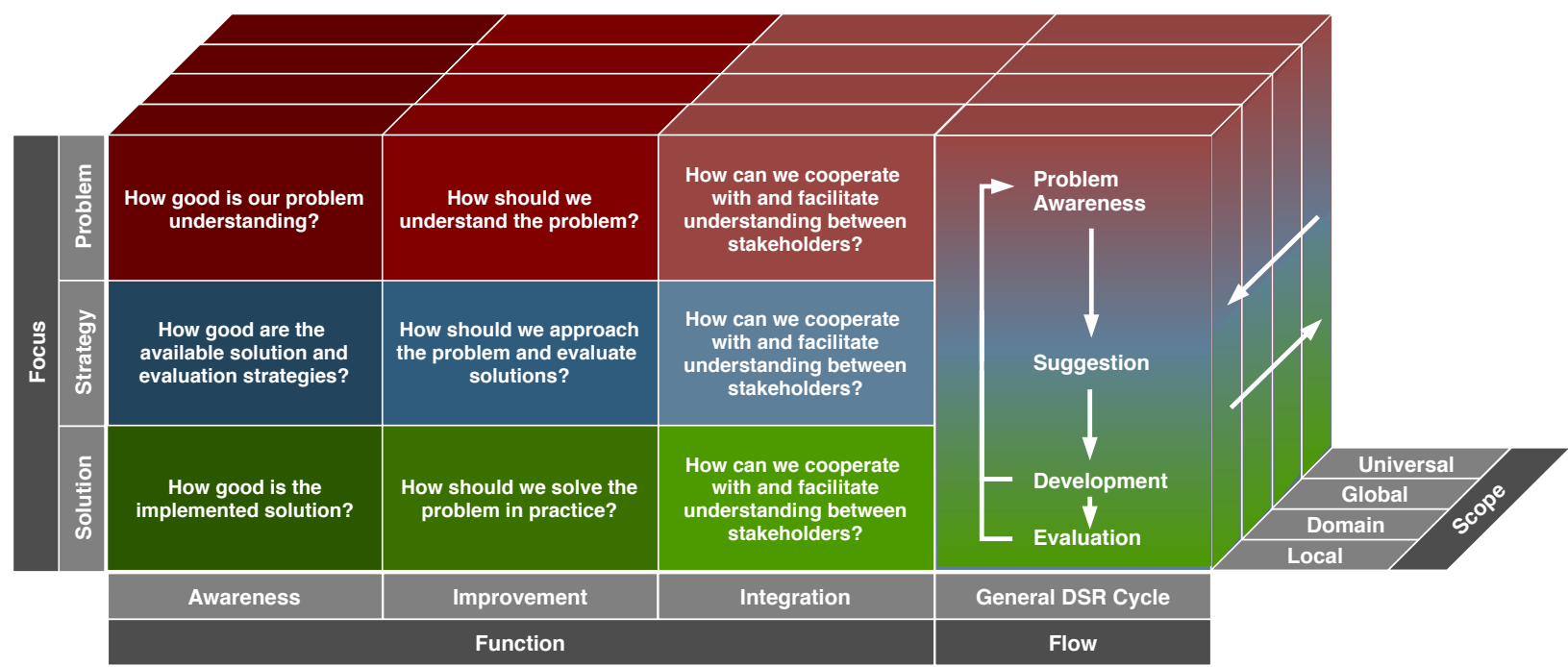

Figure 2: Context framework for DSR. The framework integrates key dimensions of our context taxonomy with the well-known general DSR cycle [3] to help design science researchers appreciate when particular context configurations are likely to be most relevant and useful.

as it flows through the general DSR cycle in a cyclical, nested, and multi-level manner [3]. Importantly, while we suggest that these context configurations are likely to be most relevant and useful at specific stages of the general DSR cycle, we want to emphasize that their positioning is not meant to be interpreted as precise. As the use of gradients in Figure 2 aims to illustrate, the 36 context configurations are likely to somewhat overlap and support each other as projects flow through the DSR cycle. Thus, it might be most fruitful to think of them as stereotypical for a specific stage of the DSR cycle. The other six dimensions of the context taxonomy cannot be systematically related to the DSR cycle in the same way and should be seen as specific to a particular phenomenon and situation at hand.

In the following we are discussing Figure 2 by rows. We suggest that, starting in the problem awareness stage of the general DSR cycle, a DSR project or stream should ideally consider all three functions of context as well as consider context across all scopes before moving on to the next stage [2,6]. This leads to 12 stereotypical context configurations with a problem focus.

First, the combination of a problem focus and the awareness function leads to a set of context configurations that is concerned with the guiding question: How good is our problem understanding? In these context configurations, the goal is to get awareness about how well-understood and represented a problem is from all relevant perspectives (e.g., ethical, social, economic, environmental, etc.). This is evaluated in terms of consensus and should ideally be done for each context scope as the nature of the problem may change depending on the boundaries that are drawn [39].

Second, the combination of a problem focus and the improvement function suggests a set of context configurations aimed at the guiding question: How should we understand the problem? In these context configurations, the goal is to improve the status quo by developing and evaluating a more useful problem representation that effectively corresponds to the underlying problem situations in question (i.e., the problem representation is insightful [40]). Again, this should ideally be done for each context scope to understand the problem in depth [41].

Third, the combination of a problem focus and the integration function sets up a set of context configurations directed at the guiding question: How can we cooperate with and facilitate understanding between stakeholders? In these context configurations, the goal is to build on and align with the work of others as well as integrate ones own contributions in a way that promotes coherence. Again, this should ideally be done for each context scope to encourage cooperation at all levels and enhance the potential impact of contributions. For instance, disseminating improved problem framings to important stakeholders in domain or even global scopes could help to spark investigations into innovative solution approaches.

In the suggestion stage of the general DSR cycle, a context focus on strategy is likely to become more predominant. Again, in this stage a DSR project or stream should ideally consider all context functions and 
scopes before moving on to the next stage [2, 6]. This leads to the identification of 12 stereotypical context configurations with a strategy focus.

First, the combination of a strategy focus and the awareness function leads to a set of context configurations that is concerned with the guiding question: How good are the available solution and evaluation strategies? In these context configurations, the goal is to get awareness about the expected cost-effectiveness and potential risks and unintended consequences of possible strategies evaluated in terms of consensus by affected stakeholders. This should ideally be done for each context scope as the availability of resources and priorities for allocating them may change depending on the chosen scope [6, 39].

Second, the combination of a strategy focus and the improvement function suggests a set of context configurations aimed at the guiding question: How should we approach the problem and evaluate solutions? In these context configurations, the goal is to improve our plans for solving the problem by identifying cost-effective solution and evaluation strategies that correspond to the particularities of the problem and situation. Again, this should ideally be done for each context scope as the best solution and evaluation strategies likely depend on the scope considered [39].

Third, the combination of a strategy focus and the integration function sets up a set of context configurations directed at the guiding question: How can we cooperate with and facilitate understanding between stakeholders? In these context configurations, the goal is to build on and align with the work of others as well as integrate ones own contributions in a way that promotes coherence. Again, this should ideally be done for each context scope to encourage cooperation at all levels and enhance the potential impact of contributions. For instance, disseminating potential solution strategies to important stakeholders in domain or even global scopes could help to direct the flow of resources to the most promising projects.

In the development and evaluation stages of the general DSR cycle, a context focus on the solution is likely to become central. We combine the development and evaluation stages of the general DSR cycle because we agree with seminal characterizations of DSR that highlight the tight interdependence between both activities [1-3]. Going further, we suggest that development should not be done without considering evaluation and evaluation should not be done without consideration of further development [42]. Again, in this stage a DSR project or stream should ideally consider all context functions and scopes to reach a comprehensive understanding [2,6]. This leads to the identification of 12 stereotypical context configurations with a solution focus.

First, the combination of a solution focus and the awareness function leads to a set of context configurations that is concerned with the guiding question: How good is the implemented solution? In these context configurations, the goal is to get awareness about the actual positive and negative consequences of solutions evaluated in terms of consensus by all affected stakeholders. This should ideally be done for each context scope as particular solutions may have unintended consequences at different levels of analysis ${ }^{6}$

Second, the combination of a solution focus and the improvement function suggests a set of context configurations aimed at the guiding question: How should we solve the problem in practice? In these context configurations, the goal is to improve the world by developing and evaluating effective solutions that work in practice so that correspondence between the actual and desired states of the world is achieved. Again, this should ideally be done for each context scope as solving problems in practice is likely best achieved through coordinated efforts across scopes [6].

Third, the combination of a solution focus and the integration function sets up a set of context configurations directed at the guiding question: How can we cooperate with and facilitate understanding between stakeholders? In these context configurations, the goal is to build on and align with the work of others as well as integrate ones own contributions in a way that promotes coherence. Again, this should ideally be done for each context scope to encourage cooperation at all levels and enhance the potential impact of contributions. For instance, disseminating knowledge about developed solutions to practitioners or other important stakeholders in domain or even global scopes could help to scale up their reach and impact.

\section{Discussion and concluding remarks}

Our paper contributes a well-grounded, coherent and extensible framework for thinking about and dealing with context in DSR. In particular, our work provides a sound theoretical foundation for thinking about context that is rooted in pragmatism and, thus, compatible with extant perspectives on DSR [2, 17]. Importantly, we highlighted how pragmatic thinking requires analytical goals to meaningfully separate a phenomenon from its context [22, 26].

Furthermore, we explicate how good DSR requires a certain sophistication regarding the flexibility and

\footnotetext{
${ }^{6}$ For example, consider the unintended consequences of social media platforms on epistemic security on a global scale.
} 
adaptiveness in how context is constructed and evaluated. Specifically, demands on context analyses shift as the general DSR cycle progresses [3] and multiple epistemic perspectives ought to be considered along each stage [2,34]. This perspective complements prior work which has already highlighted that DSR undergoes shifts in ontological stance as a project progresses from focusing on problems to solutions [35].

Moreover, we highlight the multi-leveled nature of DSR in terms of the scope that is considered. Prior work has emphasized that DSR projects also need to be considered in relation to the context of the overarching DSR stream in the domain scope [3]. We extend this observation and suggest that the state of the world in a global scope also provides important context that ought to be considered in the advancement of DSR projects or streams [6]. All of this highlights how DSR studies should always strive to adequately consider context at all scopes and throughout all life-cycle phases.

Our results help design science researchers to do this more effectively and efficiently. In particular, we argue that our taxonomy can improve design science researchers effectiveness as it enables a better understanding of the breadth of dimensions along which context analyses might be configured as well as aids in the construction of more appropriate context analyses. For instance, our context framework illustrates what context configurations are likely to be most relevant at specific stages of DSR projects or DSR streams.

Together with the other dimensions of our taxonomy, researchers now have a checklist of important considerations that can inform the planning or evaluation of their investigations. For example, looking at the dimension of time awareness researchers are encouraged to consciously reflect whether a dynamic perspective on time may be needed or if a static perspective is adequate given the problem under investigation.

The dimensions can also help to structure and improve the documentation of research activities and facilitate higher quality peer-review as it becomes easier to point to specific aspects that ought to be discussed as part of rigorous scientific publications.

We argue that our work can also improve efficiency as it integrates a sophisticated understanding of context into a format that is easier and quicker to understand and use than the alternative-time-consuming engagement with the philosophical peculiarities of context.

In terms of limitations of our work, we acknowledge that we have not yet rigorously evaluated the utility of the taxonomy and framework in applied empirical settings beyond personal use by the authors. While we have outlined the potential usefulness of our work, we recognize that all DSR outcomes should ideally be evaluated in a more rigorous manner [2]. Thus, we encourage other design science researchers to report on the usefulness of our taxonomy and framework in the context of their own research or, even better, to test it experimentally.

Future work is also encouraged to adapt our results and further develop them in novel ways. For instance, our taxonomy could provide a useful starting point for the development of a community-oriented research support system where information about the context of research activities is systematically collected and then used as a basis for a context-driven recommender system to improve the efficiency of DSR [43]. Altogether, our work aspires to provide a sound foundation and a fruitful platform for future research to more deeply engage with context in DSR.

\section{Acknowledgements}

This research was partly funded by the German Federal Ministry for Economic Affairs and Energy (BMWi), project FLEMING (03E16012F).

\section{References}

[1] S. Gregor and A. R. Hevner, "Positioning and Presenting Design Science Research for Maximum Impact," MIS Quarterly, vol. 32, no. 2, pp. 337-355, 2013.

[2] A. R. Hevner, S. T. March, J. Park, and S. Ram, "Design Science In Information Systems Research," MIS Quarterly, vol. 28, no. 1, pp. 75-105, 2004.

[3] V. Vaishnavi and W. J. Kuechler, Design Science Research Methods and Patterns. CRC Press, 2nd ed., 2015.

[4] J. vom Brocke, R. Winter, A. R. Hevner, and A. Maedche, "Accumulation and Evolution of Design Knowledge in Design Science Research - A Journey Through Time and Space," Journal of the AIS, vol. 21, no. 3, pp. 520-544, 2020.

[5] R. Gholami, R. Watson, H. Hasan, A. Molla, and N. Bjorn-Andersen, "Information Systems Solutions for Environmental Sustainability: How Can We Do More?," Journal of the AIS, vol. 17, no. 8, pp. 521-536, 2016.

[6] A. Herwix and A. Haj-Bolouri, "Having a positive impact with Design Science Research - Learning from Effective Altruism," in DESRIST 2020, (Kristiansand, Norway), 2020.

[7] M. Jirotka, B. Grimpe, B. Stahl, G. Eden, and M. Hartswood, "Responsible research and innovation in the digital age," Communications of the ACM, vol. 60, no. 5, pp. 62-68, 2017.

[8] C. Avgerou, "Contextual explanation: Alternative approaches and persistent challenges," MIS Quarterly, vol. 43 , no. 3 , pp. 977-1006, 2019.

[9] R. M. Davison and M. G. Martinsons, "Context is King! Considering Particularism in research design and reporting," Journal of Information Technology, vol. 31, no. 3, pp. 241-249, 2016. 
[10] Merriam-Webster, "Context." https: //merriam-webster.com/dictionary/ context. accessed 2021-05-18.

[11] A. Maedche, S. Gregor, S. Morana, and J. Feine, "Conceptualization of the Problem Space in Design Science Research," in DESRIST 2019, 2019.

[12] A. S. Lee and R. L. Baskerville, "Generalizing Generalizability in Information Systems Research," Information Systems Research, vol. 14, no. 3, pp. 221-243, 2003.

[13] Lee and Baskerville, "Conceptualizing Generalizability: New Contributions and a Reply," MIS Quarterly, vol. 36, no. 3 , p. $749,2012$.

[14] R. Baskerville and J. Pries-Heje, "Design theory projectability," in Working Conference on Information Systems and Organizations, pp. 219-232, Springer, 2014.

[15] R. Baskerville and J. Pries-Heje, "Projectability in Design Science Research," JITTA: Journal of Information Technology Theory and Application, vol. 20 , no. 1,2020 .

[16] M. G. Martinsons and R. M. Davison, "People, Places and Time in Research Design and Reporting: Responding to Commentaries on Particularism," Journal of IT, vol. 31, pp. 267-268, Sept. 2016

[17] G. Goldkuhl, "Design Science Epistemology: A pragmatist inquiry," Scandinavian Journal of Information Systems, vol. 32, no. 1, pp. 39-80, 2020.

[18] R. C. Nickerson, U. Varshney, and J. Muntermann, "A method for taxonomy development and its application in information systems," European Journal of Information Systems, vol. 22, no. 3, pp. 336-359, 2013.

[19] P. Bamberger, "From the Editors Beyond Contextualization: Using Context Theories to Narrow the Micro-Macro Gap in Management Research," Academy of Management Journal, vol. 51, no. 5, pp. 839-846, 2008.

[20] K. D. Elsbach and M. G. Pratt, "The Physical Environment in Organizations," Academy of Management Annals, vol. 1, no. 1, pp. 181-224, 2007.

[21] N. Hayes and C. Westrup, "Context and the processes of ICT for development," Information and Organization, vol. 22, no. 1, pp. 23-36, 2012.

[22] S. C. Hayes, L. J. Hayes, and H. W. Reese, "Finding the philosophical core: a review of Stephen C. Pepper's world hypotheses: a study in evidence," Journal of the experimental analysis of behavior, vol. 50, no. 1, pp. 97-111, 1988 .

[23] G. Johns, "The Essential Impact of Context on Organizational Behavior," Academy of Management Review, vol. 31, no. 2, pp. 386-408, 2006.

[24] D. M. Rousseau and Y. Fried, "Location, location, location: Contextualizing organizational research," Journal of organizational behavior, pp. 1-13, 2001.

[25] T. J. Scheff, "The Structure of Context: Deciphering Frame Analysis," Sociological Theory, vol. 23, no. 4, pp. 368-385, 2005.

[26] R. D. Zettle, S. C. Hayes, D. Barnes-Holmes, and A. Biglan, The Wiley Handbook of Contextual Behavioral Science. John Wiley \& Sons, 2016.
[27] B. Latour, Reassembling the Social: An Introduction to Actor-Network-Theory. OUP Oxford, 2005.

[28] W. J. Orlikowski and S. V. Scott, "Sociomateriality: Challenging the Separation of Technology, Work and Organization," The Academy of Management Annals, vol. 2, no. 1, pp. 433-474, 2008.

[29] K. Barad, Meeting the Universe Halfway. Duke University Press, 2007.

[30] S. Gregor, L. Chandra Kruse, and S. Seidel, "The Anatomy of a Design Principle," Journal of the AIS, 2020.

[31] A. Biglan, D. Ary, and A. C. Wagenaar, "The Value of Interrupted Time-Series Experiments for Community Intervention Research," Prevention Science, vol. 1, no. 1, pp. 31-49, 2000.

[32] X. Liu, K. Werder, and A. Maedche, "Novice digital service designers' decision-making with decision aids A comparison of taxonomy and tags," Decision Support Systems, vol. 137, p. 113367, 2020.

[33] J. Webster and R. Watson, "Analyzing the past to prepare for the future: Writing a literature review," $M I S Q$., vol. 26, 2002.

[34] J. Mingers and C. Standing, "A Framework for Validating Information Systems Research Based on a Pluralist Account of Truth and Correctness," Journal of the AIS, vol. 21, no. 1, 2020.

[35] S. Purao, "Truth or dare: The ontology question in design science research," Journal of Database Management (JDM), vol. 24, no. 3, pp. 51-66, 2013.

[36] S. Sarker, S. Chatterjee, Xiao Xiao, and A. Elbanna, "The Sociotechnical Axis of Cohesion for the Is Discipline: Its Historical Legacy and Its Continued Relevance," MIS Quarterly, vol. 43, no. 3, pp. 695-A5, 2019.

[37] L. Chandra Kruse, S. Seidel, and J. v. Brocke, "Design archaeology: Generating design knowledge from real-world artifact design," in DESRIST 2019, 2019.

[38] J. Venable, J. Pries-Heje, and R. Baskerville, "Feds: a framework for evaluation in design science research," European Journal of Information Systems, vol. 25, no. 1, pp. 77-89, 2016.

[39] W. Ulrich, "Critical Pragmatism: A New Approach to Professional and Business Ethics," in Interdisciplinary Yearbook for Business Ethics. v. 1, v. 1, Bern: Peter Lang Pub Inc, 2006.

[40] J. Vervaeke and L. Ferraro, "Relevance, Meaning and the Cognitive Science of Wisdom," in The Scientific Study of Personal Wisdom (M. Ferrari and N. M. Weststrate, eds.), pp. 21-51, Dordrecht: Springer Netherlands, 2013.

[41] A. Herwix and A. Haj-Bolouri, "Revisiting the Problem of the Problem - An Ontology and Framework for Problem Assessment in IS Research," in Proceedings of the Twenty-Nineth European Conference on Information Systems (ECIS2021), 2021.

[42] D. L. Stufflebeam and C. Coryn, Evaluation Theory, Models, and Applications. Hoboken, N.J.: John Wiley \& Sons, 2014

[43] R. Pagano, P. Cremonesi, M. Larson, B. Hidasi, D. Tikk, A. Karatzoglou, and M. Quadrana, "The Contextual Turn: from Context-Aware to Context-Driven Recommender Systems," in Proceedings of the 10th ACM Conference on Recommender Systems, RecSys '16, (New York, NY, USA), pp. 249-252, Association for Computing Machinery, 2016. 